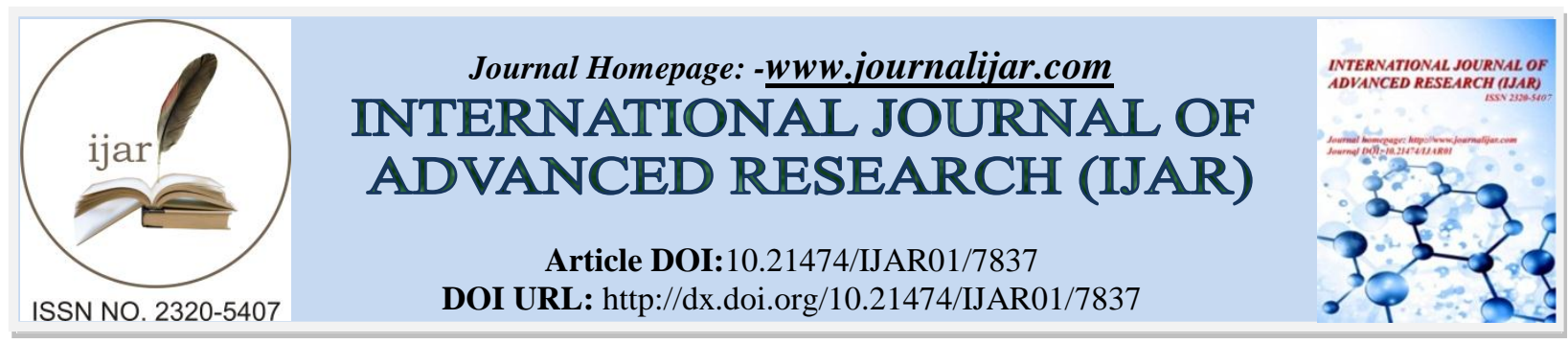

RESEARCH ARTICLE

\title{
DESIGN AND DEVELOPMENT OF FLUSH AIR DATA SENSING (FADS) SYSTEM FOR WINGED ROCKET.
}

Guna Surendra Gossamsetti, Koichi Yonemoto and Takahiro Fujikawa.

Department of Mechanical and Control Engineering, Kyushu Institute of Technology, Japan.

\section{Manuscript Info}

\section{Manuscript History}

Received: 7 August 2018

Final Accepted: 9 September 2018

Published: October 2018

Keywords:-

FADS, Re-entry, Winged Rocket..

\begin{abstract}
The primary goal of the Single-Stage-to-Orbit technology is radially to reduce the cost of access to Space. The Winged rocket developed by Kyushu Institute of Technology, Japan is the test bed and technology demonstrator for this effort. To successfully complete these missions, estimation of air data parameters are vital. FADS (Flush Air Data System) is utilized for the winged rocket. The flight control system requires indispensable air data acquisition with high precision and high reliability. However, under severe thermal environment of re-entry, the failure risk of pressure ports is high and there is as possibility that acquisition of the air data accuracy may not be reliable, which adversely affects the flight control. In this paper, the improved design of Flush Air Data Sensing (FADS) system is proposed for estimation of air data parameters for wide flight range. Wind tunnels tests have been performed for the calibration and calculating the algorithm of FADS. However, the measured and estimated values had little discrepancy in accuracy. Statistical accuracy of the calibration is evaluated by comparing the wind tunnel reference conditions to the air data parameters estimated. This comparison is accomplished by applying the calibrated FADS algorithm to the sensed wind tunnel pressures.
\end{abstract}

Copy Right, IJAR, 2018,. All rights reserved.

\section{Introduction:-}

The primary goal of Single-Stage-to-Orbit technology is to radically reduce the cost of transportation to and from space. Already, many private organizations are developing reusable space planes for commercial use. Since 2005, the Kyushu Institute of Technology, Japan has been developing a suborbital winged rocket called WIRES (WInged Reusable Sounding rocket) for use in research projects of future, fully reusable space transportation. To successfully complete these missions, estimation of air data parameters are vital. Conventional pitot tubes are not suitable for operation in hypersonic flight environments due to extreme heating of the Pitot tube caused by its small radius. Also, the shock and expansion wave created by conventional pitot tubes have a damaging effect on the spacecraft, hence the concept of FADS, in which the air data is inferred from nonintrusive surface pressure measurements (Figure 1). This innovation allows for the continued operation of instrumentation during high temperature, supersonic re-entry, which extends the useful range of the air data measurement system to hypersonic flow regime. FADS (Flush Air Data System) is also utilized by the authors for estimation of the air data parameters on WIRES, whose flight control system requires air data acquisition with high precision and high reliability. 

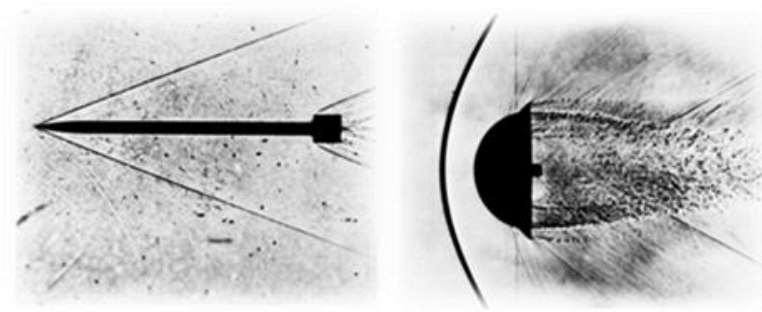

Figure 1:- Shock wave propagation on sharp and blunt object

However, in the extreme thermal environment of re-entry, pressure ports still have a high risk of failure, making the acquisition of the air data unreliable and adversely affecting flight control. From the 1980s, NASA has been studying the implementation of FADS for hypersonic vehicles such as the X-15 and X-33 ${ }^{1}{ }^{2}$. JAXA (Japan Aeronautical Exploration Agency) had also been studying the implementation of FADS in a hypersonic vehicle called HYFLEX (HYpersonicFLightEXperiment), but was never practically implemented due to the cancellation of the project. In addition, due to the severe conditions during re-entry, the failure risk of the measuring apparatus was even higher. Therefore, by utilizing a large number of pressures holes and developing FADS with fault tolerance design, the authors aim to create a system that is able to operate in any environment.

Recently, Todaka et al. proposed an algorithm based on Tashiro's method of calculating static pressure ${ }^{3)}$ and referred to Suenaga's method to calculate the calibration factor ${ }^{4)}$ for a FADS specimen. The calibration term is defined by the static pressure and pressure coefficients, which are calculated from the measured pressures of 17 different ports for the redundancy. However, Mach number estimation results showed discrepancies, therefore the authors adopted new algorithm based on the X-33's method. In this paper, the design specimen of FADS, transonic wind tunnel experiment results, and air data estimation results using the X-33's method are discussed. FADS mounted on a next generation winged rocket (Figure 2) developed at the Kyushu Institute of Technology are also discussed.

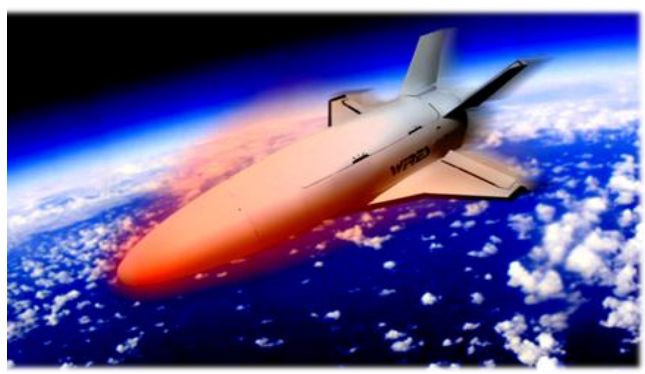

Figure 2:- Artist rendering of winged rocket during re-entry

\section{Wind Tunnel Experiment Experimental apparatus}

Pressure distribution measurements are performed using the transonic wind tunnel located at the JAXA Sagamihara campus in Japan (Figure 3). The shape of the FADS specimen is designed according to shape of the nose cap of the winged rocket. The specifications of the specimen are shown in the Figure 4. The frontal diameter of the specimen is $110 \mathrm{~mm}$ and the radius of curvature of the specimen tip is $43 \mathrm{~mm}$, with 17 pressure sensing holes. This 17 measurements slot configurations is the initial design and is based on previous FADS model. The number of measurements ports selected is a compromise between the need to accurately measure the flow condition at the nose and the cost of manufacture of the ports on the vehicle. 


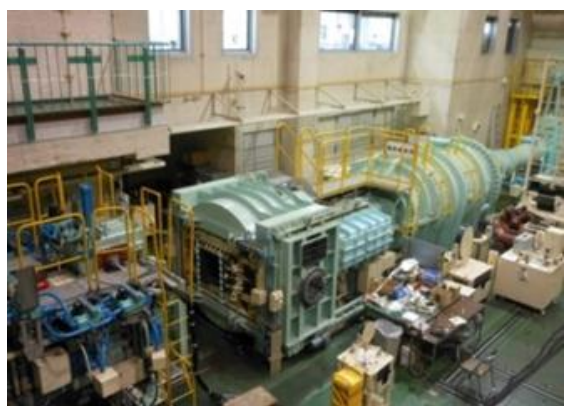

Figure 3:- Hypersonic wind tunnel at JAXA Sagamihara
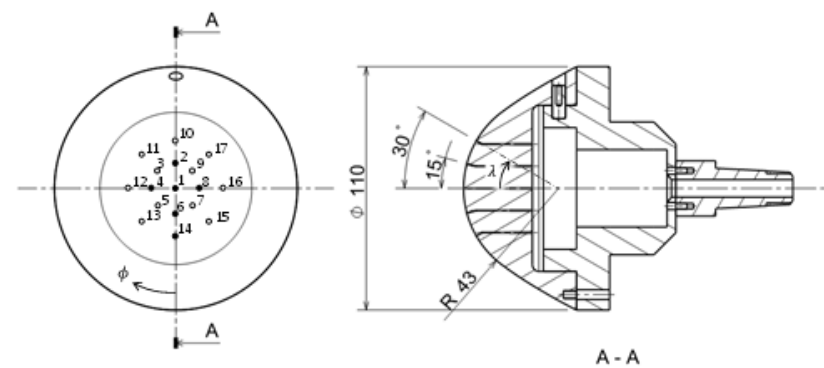

Figure 4:- FADS specimen drawing

\section{Test conditions}

The specimen is tested according to the test conditions in Table 1. The experimental data of the FADS for these test conditions have been analyzed using the algorithm discussed below.

Table 1:-Test conditions

\begin{tabular}{|c|c|}
\hline Mach number & $0.3,0.5,0.7,0.8,0.9,1.0$ \\
\hline Angle of attack & $-4^{\circ} \sim 20^{\circ}\left(\right.$ every $\left.2^{\circ}\right)$ \\
\hline Sideslip angle & $0^{\circ} \sim 4^{\circ}\left(\right.$ every $\left.2^{\circ}\right)$ \\
\hline
\end{tabular}

\section{Air Data Estimation Algorithm}

\section{Aerodynamic model}

The fundamental concept of the FADS system is that air data parameters can be estimated from the flush surface pressure measurements. To perform this estimation, the air data must be related to the surface pressures by an aerodynamic model that accurately models the flow and is valid over a large Mach number range. The aerodynamic model is derived from the closed form potential flow solution for blunt bodys which is applicable at subsonic speeds and the modified Newtonian flow model applicable at hypersonic speeds. Both the potential flow and the Newtonian flow are described as measured pressure coefficient in terms of the incident angle. To blend the two solutions over a large range of Mach numbers, a calibration parameter is introduced. The resulting model Equation (1) is as follows.

$$
p_{i}=q_{c}\left[\cos ^{2} \theta_{i}+\varepsilon \sin ^{2} \theta_{i}\right]+p_{\infty}
$$

The incident angle is related to the local or effective angle of attack and sideslip angle as shown in the following.

$$
\cos \left(\theta_{i}\right)=\cos \left(\alpha_{e}\right) \cos \left(\beta_{e}\right) \cos \left(\lambda_{i}\right)+\sin \left(\beta_{e}\right) \sin \left(\phi_{i}\right) \sin \left(\lambda_{i}\right)+\sin \left(\alpha_{e}\right) \cos \left(\beta_{e}\right) \cos \left(\phi_{i}\right) \sin \left(\lambda_{i}\right)
$$

In Equation (2), the cone angle is the total angle the normal surface makes with respect to the longitudinal axis of the nose cap. The clock angle is the clockwise angle looking around the axis of the symmetry starting at the bottom of the fuselage. These coordinate angles are shown in the Figure 4. The remaining parameters in Equation (1) are impact pressure and free stream static pressure. Using these four basic parameters, most of the other air data can be calculated. 


\section{Port selection for the algorithm}

Since there are four air data states and a calibration parameter to be estimated, at least five independent pressure measurements must be available to derive the entire air data state. Using five sensors to estimate the air data is equivalent to a high order spline fit and results in an estimating which is sensitive to noise in the measured pressures. Providing an additional sixth sensing location mitigates the noise sensitivity, increases redundancy options, and results in a system which gives overall superior performance. Figure 5 shows the FADS measurement locations. The layout along the meridian lines allows the calculation for angle of attack to be decoupled from sideslip angle calculations. The system layout is designed to be sensitive to angles of attack varying from -20 to 45 , and angles of sideslip up to +-20 .

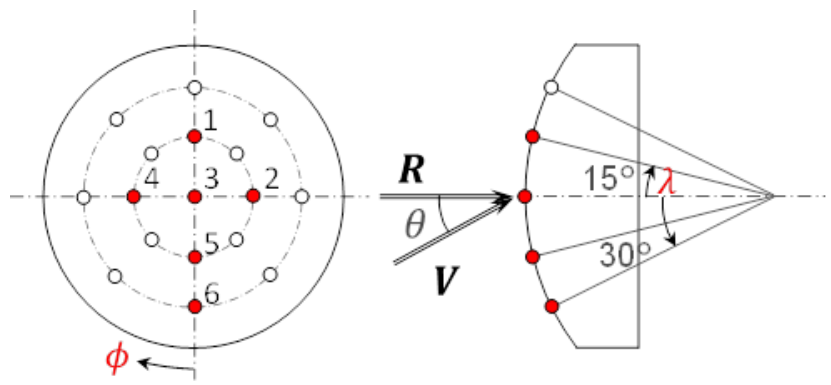

Figure 5:- FADS measurement locations

Table 2:-FADS ports' clock and cone angles

\begin{tabular}{|c|c|c|}
\hline Port No. & $\phi_{i}\left[{ }^{\circ}\right]$ & $\lambda_{i}\left[{ }^{\circ}\right]$ \\
\hline 1 & 180 & 15 \\
\hline 2 & 270 & 15 \\
\hline 3 & 0 & 0 \\
\hline 4 & 90 & 15 \\
\hline 5 & 0 & 15 \\
\hline 6 & 0 & 30 \\
\hline
\end{tabular}

\section{Flow chart for the algorithm}

The air data parameters are estimated from Equation (1) and Equation (2) however, for a local angle of attack and sideslip angle, combinations of three pressure holes are strategically selected. Then the relationship between static, impact pressures and calibration coefficient is eliminated. The flow chart for the estimation of parameters is shown in Figure 6 and 7. The algorithm has two segments; one for estimation of calibration coefficient and the other for the estimation of other physical parameters. Using the wind tunnels results, the calibration coefficient is determined while the air data parameters are estimated for the actual flight test.

$$
P_{i} \longrightarrow \alpha_{e} \longrightarrow \beta_{e} \longrightarrow \theta_{i} \longrightarrow \varepsilon \longrightarrow \delta \alpha \longrightarrow \delta \beta
$$

Figure 6:- FADS calibration flowchart

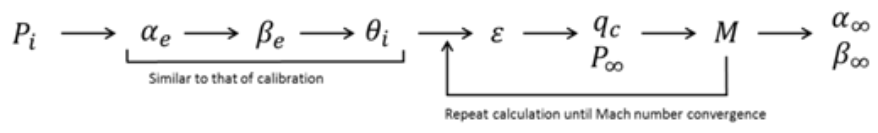

Figure 7:- FADS estimation flowchart

\section{Calibration coefficientcalculationalgorithm \\ Estimation of local angle of attack}

By strategically taking the differences of three surface pressures called triples (Figure 8), the pressure related states are eliminated from Equation (1). The resulting pressure equation is shown in Equation 3.
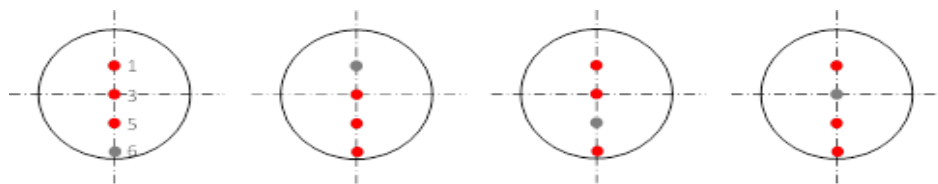

Figure 8:- Combination of pressure holes for angle of attack estimates 


$$
\begin{aligned}
\Gamma_{i k} \cos ^{2} \theta_{j} & +\Gamma_{j i} \cos ^{2} \theta_{k}+\Gamma_{k j} \cos ^{2} \theta_{i}=0 \\
\Gamma_{i k} & =\left(p_{i}-p_{k}\right) \\
\text { where, } \Gamma_{j i} & =\left(p_{j}-p_{i}\right) \\
\Gamma_{k j} & =\left(p_{k}-p_{j}\right)
\end{aligned}
$$

Here in Equation (3), the local angle of attack is decoupled from the local sideslip angle using only pressures aligned along a vertical meridian of $=0^{\circ}$ and $180^{\circ}$. The local angle of attack solution is shown in Equation (4) and Equation (5). The output of the angle of attack is determined as mean of the values computed using four individual triples. The average of the results provides a measure of noise rejection for the estimator. If one of ports along the vertical meridian is deemed unusable and it is weighted out of the algorithm, then only one valid triple remains for computing the angle of attack.

$$
\begin{aligned}
& A\left(\tan ^{2} \alpha_{e}-1\right)+2 B \tan \alpha_{e}=0 \\
& \hat{\alpha}_{e}=\left.\frac{1}{n} \sum_{x=1}^{n} \hat{\alpha}_{e x}\left(p_{\{i, j, k\}}, \lambda_{\{i, j, k\}}, \phi_{\{i, j, k\}}\right)\right|_{n=4}
\end{aligned}
$$

Estimation of local sideslip angle

Once the angle of attack is estimated, then the sideslip angle may be evaluated using any combination of the available ports that lie on the vertical meridian. The result for estimating the local sideslip angle is shown below.

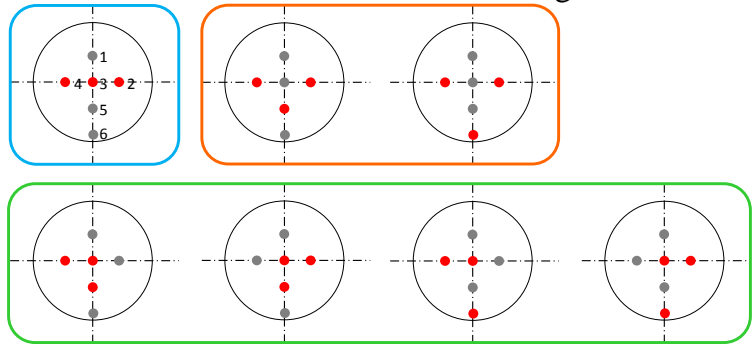

Figure 9:- Combination of pressure holes for sideslip angle estimates

$$
\begin{aligned}
& A^{\prime} \tan ^{2} \beta_{e}+2 B^{\prime} \tan \beta_{e}+C^{\prime}=0 \\
& \hat{\beta}_{e}=\left.\frac{1}{n} \sum_{x=1}^{n} \hat{\beta}_{e x}\left(p_{\{i, j, k\}}, \lambda_{\{i, j, k\}}, \phi_{\{i, j, k\}}, \hat{\alpha}_{e}\right)\right|_{n=7}
\end{aligned}
$$

The output of the sideslip estimate is determined in the same manners as the angle of attack (Equation $(6,7)$ ). It is the mean of the values computed using the four individual triples. Again, if one of the ports is deemed unusable and weighted out of the algorithm, then only one valid triple remains for the computing the sideslip angle.

\section{Estimation of incidence angle}

The incidence angle for the respective local angle of attack and sideslip angle at the respective ports with cone and clock angles are estimated using Equation (3-2).

\section{Calculation of calibration factor}

Once the angle attack and sideslip angle are determined, the incident angle at each pressure hole is calculated using Equation (8). In addition, the calibration factor is formulated using the pressure field model from Equation (9) as shown below. 


$$
\begin{aligned}
& \frac{p_{i}\left(\theta_{i}\right)-P_{\infty}}{q_{c}}=\cos ^{2} \theta_{i}+\varepsilon \sin ^{2} \theta_{i} \\
& C_{p c}\left(\theta_{i}\right) \equiv \frac{p_{i}\left(\theta_{i}\right)-P_{\infty}}{q_{c}} \\
& =\frac{p_{i}\left(\theta_{i}\right)-P_{\infty}}{(\text { stagnation point pressure })-P_{\infty}} \\
& {\left[\begin{array}{c}
C_{p_{c}}\left(\theta_{1}\right)-\cos ^{2}\left(\theta_{1}\right) \\
\vdots \\
C_{p_{c}}\left(\theta_{n}\right)-\cos ^{2}\left(\theta_{n}\right)
\end{array}\right]=\left[\begin{array}{c}
\sin ^{2}\left(\theta_{1}\right) \\
\vdots \\
\sin ^{2}\left(\theta_{n}\right)
\end{array}\right] * \varepsilon\left[M_{\infty}, \alpha_{e}, \beta_{e}\right]} \\
& \varepsilon\left[M_{\infty}, \alpha_{e}, \beta_{e}\right]=\frac{\sum_{i=1}^{n} \sin ^{2}\left(\theta_{i}\right)\left[C_{p_{c}}\left(\theta_{i}\right)-\cos ^{2}\left(\theta_{i}\right)\right]}{\sum_{i=1}^{n} \sin ^{4}\left(\theta_{i}\right)}
\end{aligned}
$$

\section{Main stream angle of attack and sideslip angle}

The FADS is also used to estimate the local angle of attack and side slip angle on the blunt shape, which is different from the mainstream angle of attack and sideslip angle and can be derived for a winged rocket using the calibration factor. The calibration formula is derived using the reference values from the wind tunnel experiments. Estimated values are calculated using the formulas shown in Equation (10) and Equation (11).

$$
\begin{aligned}
& \delta \alpha=\alpha_{e}-\alpha_{r e f} \\
& \delta \beta=\beta_{e}-\beta_{r e f}
\end{aligned}
$$

\section{Physical quantity estimation algorithm}

The FADS measures the total pressure at all ports and, using the calibration coefficient $\varepsilon$ calculated in section 3.4.3, calculates air data parameters (angle of attack, side slip angle and Mach number) according to Figure 9.

\section{Estimation of local angle of attack, sideslip angle and incidence angle}

The same method of calculation (Equation 1 to Equation 7) is used for the calculation of the local angle of attack, sideslip angle and incidence angle using the total pressure data from each port of the FADS.

\section{Mach number estimation}

Once the values of estimated angle of attack and sideslip angle have been determined, then the incidence angle at all ports can be evaluated and only calibration factor and freestream static pressure remains as unknowns in the pressure equations. As calibration factor is implicitly a function of static pressure and dynamic pressure, the resulting system of equations is nonlinear. The solutions for static pressure and dynamic pressure must be extracted iteratively. The calibration factor is a function of Mach number and is used for estimation of the Mach number.

For deriving the Mach number iteratively, the calibration coefficient for the suitable angle of attack is required. Based on the calibration coefficient results from the wind tunnel, Mach numbers for the given local angle of attack is obtained. The initial Mach is obtained and the calculation is repeated iteratively until the Mach number converges. Mach numbers can be computed using normal, one dimensional fluid mechanics relationships. Sub-sonically, Mach numbers can be calculated directly using isentropic flow laws is shown in Equation (12). Supersonically, the solution is computed using the Rayleigh pitot equation, which is derived from adiabatic normal shock relationships and is shown in Equation (13).

$$
\frac{q_{c}}{P_{\infty}}=\left(1+\frac{\kappa-1}{2} M_{\infty}^{2}\right)^{\kappa / \kappa-1}-1
$$




$$
\frac{q_{c}}{P_{\infty}}=\left(\frac{(\kappa-1) M_{\infty}^{2}}{2}\right)^{\kappa / \kappa-1}\left(\frac{\kappa-1}{2 \kappa M_{\infty}^{2}-(\kappa-1)}\right)^{1 / \kappa-1}-1
$$

\section{Results and Conclusion:-}

A wind tunnel test at transonic range is performed on the FADS specimen developed for the winged rocket to calculate the air data parameters for a range of test conditions. The calibration coefficient estimation results for various Mach numbers and angles of attack are shown in the Figure 10. It is understood that the calibration coefficient is a function of the local angle of attack and Mach number. Based on the experimental data from the wind tunnel, the local angle of attack and sideslip angle are estimated in the transonic region. The estimates of the calculations at their respective Mach number are shown in the Figure 11 and Figure 12. From the results it is clearly evident that the angle of attack has no dependency on the Mach number but the sideslip angle does. Also when the sideslip angle increases, the estimate is affected non-linearly.

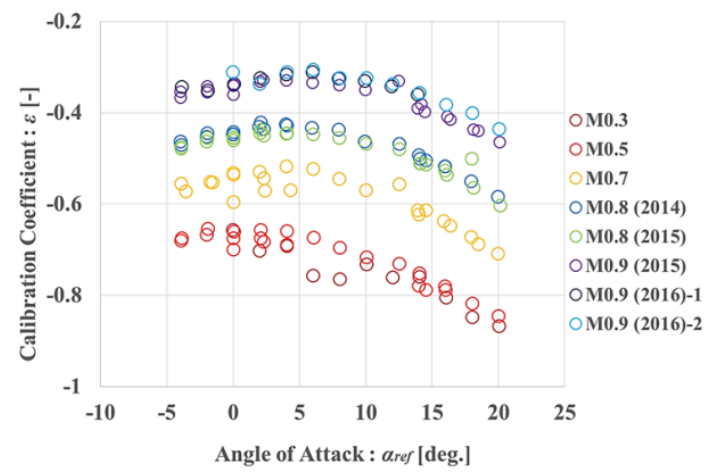

Figure 10:-Calibration coefficient estimation results

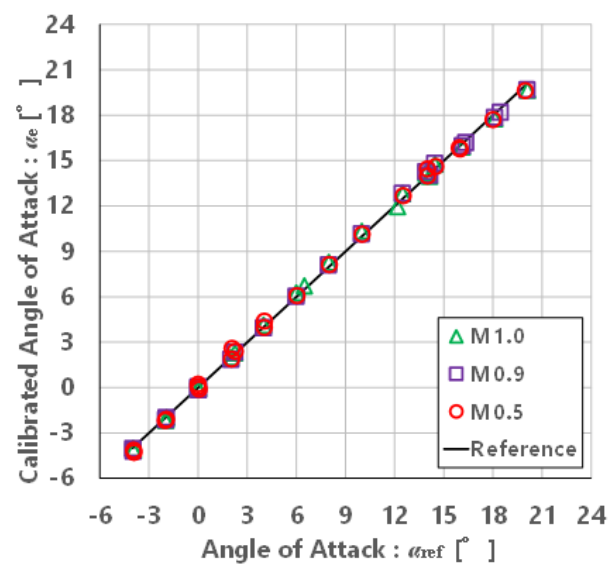

Figure11:-Free stream angle of attack estimation results

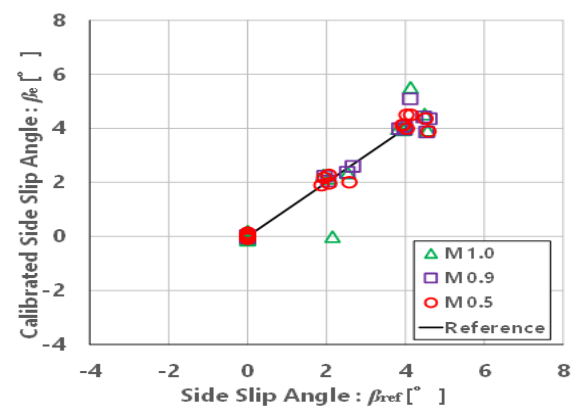

Figure 12:- Free stream sideslip angle estimation results 
The Mach number estimation results for different angle of attack and sideslip angle are calculated. The estimated angle of attack and sideslip angle were similar to that of the reference angle of attack and sideslip angle with a very small error in the transonic regions. The error analysis shown in Figure 13 concluded that the algorithm should be revised to reduce the gap between the estimated and reference values and improve the accuracy of the FADS. It is also noted that there are some singularity points which resulted in faulty values when calculating sideslip angle.

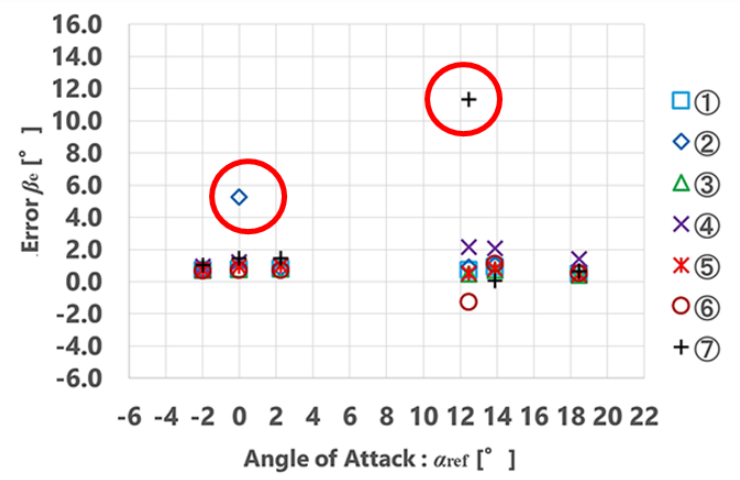

Figure 13:- Free stream sideslip angle estimation results

Currently, the authors are performing wind tunnel experiments in supersonic region ${ }^{5)}$ and also studying about the elimination of the singularity points. They are also planning to implement a fault tolerance ${ }^{6}$ capability for the estimation algorithm, as the failure risk of the measuring apparatus is high. This algorithm, if successful, would be implemented in the WIRES\#013, the current version of the winged rocket, which is expected to be launched in December 2019.

\section{Acknowledgement:-}

The authors thank Japan Aerospace Exploration Agency (JAXA) for providing wind tunnel test facility at Sagamihara campus every year to test FADS, without which the research would not have been progressed.

\section{References:-}

1. Brent R. Cobleigh et al. (1999), Flush Airdata Sensing (FADS) System Calibration Procedure and Results for Blunt Forebodies, NASA, pp.1-9.

2. Stephen A. Whitmore et al. (1998), Design and Calibration of the X-33 Flush Airdata Sensing (FADS) System, NASA, pp.1-31.

3. Tashiro Shinichi, YoshikiHaruo (1981), "Flow Measurement of Dynamic and Static Pressure Gradients by means of a Multi-hole Yaw meter", Institute of Industrial Science, University of Tokyo, 33(9), pp. 384-387.

4. SuenagaHisashi et al (2002), "Wing-tip Vortex Measurement by 7-hole Probe", National Aerospace Laboratory materials, 765, pp. 2-5.

5. John D. Anderson Jr. (2006), Hypersonic and High-Temperature Gas Dynamics Second Edition, AIAA, Virginia, pp.306-312.

6. V. VenkataSubramanian(2003), A review of process fault detection and diagnosis, Part I: Quantitative modelbased methods, Computers and Chemical Engineering 27 293/311 